\title{
BIODETERIORATION OF CHEMICAL CONSTITUENTS IN DRUG GMELINA ARBOREA LINN. UNDER STORAGE OF DIFFERENT RELATIVE HUMIDITIES DUE TO SPOILAGE OF FUNGI
}

M Rashidi* and SS Deokule

Department of Botany, University of Pune, Pune-411007- India

Received on: 12/08/12 Revised on: 20/10/12 Accepted on: 08/11/12

\author{
*Corresponding author \\ E-mail: rashidi_129@yahoo.com \\ DOI: $10.7897 / 2277-4343.03627$ \\ Published by Moksha Publishing House. Website www.mokshaph.com \\ All rights reserved.
}

\section{ABSRACT}

The roots of plant Gmelina arborea Linn. were selected for the present investigation. The roots were stored at different relative humidities $30,50,75$, 96 and $100 \% \mathrm{RH}$ for 90 days. Total 15 fungal species were isolated from these roots. Quantitative estimation of phenols, proteins, alkaloids, glycosides and sugars in relation to fungi were done. Maximum percentage incidence of storage fungi as well as deterioration of chemical constituents in the roots was showed at above $75 \% \mathrm{RH}$.

Key words: Deterioration, relative humidity, quantitative estimation, mycoflora

\section{INTRODUCTION}

Gmelina arborea is commonly called as "Shivan" or "Shewun", belonging to family "Verbenaceae". The roots contain yellow viscid oil, resin, alkaloid, a trace of benzoic acid, gmelinol, hentria contanol-1 ceryl alcohol, n- octacosanol- $\beta$ sitosteral and sesquiterp candidane type of furanoses quiterp gmelofuran and ash, free from manganese. The roots are acidic, bitter, tonic, stomachic, laxative, galactogogue, demulcent antibilious, febrifuge and anthelmintic. They are useful in hallucination, fever, dyspepsia, hyperdipsia, haemorrhoids, stomachalgia, heart diseases, nervous disorders, piles and burning sensation ${ }^{1}$ ${ }^{7,14}$. Bark is bitter, hypoglycaemic, antiviral, anticephalgic and tonic. It is used in fever and dyspepsia ${ }^{14}$. The leaves are demulcent and antigonorrhoeic. Extract of the root is bitter and tonic ${ }^{7}$.

The traditional methods of collection, storage and marketing coupled with humid climatic condition make them victim to the fungal infestation. Our this work is in continuation of earlier investigations reported on association of various fungi with herbal drugs under storage.

During storage, the fungal organisms thrive on drug plants by utilizing various components including the active ingredients and changes in active material content such as carbohydrates, alkaloids, phenol, glycosides and protein. Contents of stem, seed, fruit and root are influenced both by fungi as well as physical factors ${ }^{10,13}$. However, the information is still scanty; therefore, it was decided to investigate the isolation of mycoflora from the roots of herbal drug Gmelina arborea and changes in chemical constituents under influence of Relative humidity.

\section{MATERIAL AND METHODS}

The root samples of drug G. arborea were collected from different shopkeepers of Pune and Mumbai, then authenticated by the help of Botanical survey of India (BSI); they were brought to the laboratory in polyethylene bags to avoid other aerial contaminations. Samples were stored in small muslin clothes in desiccators at 30, 50, 75, 96 and $100 \%$ RH for 90 days in the room temperature ${ }^{15}$. The root samples were taken out on interval of 15, 30, 45, 60, 75 and 90 days, thoroughly washed with distilled water. According International Seed Testing Association ${ }^{4}$, Moist Blotter test and Agar plate method were adopted for isolation and identification of associated fungi. For isolation of associated mycoflora, small pieces of root samples sterilized with $2 \%$ sodium hypochlorite solution and thoroughly washed with $100 \mathrm{ml}$ of sterilized distilled water. After developing, colonies were counted and average of 10 Petri plates were calculated from first day to $60^{\text {th }}$ day of storage. Fungi were identified by using references such as Raper and Thom ${ }^{9}$ Barnet and Hunter ${ }^{2}$ and Nelson et al. ${ }^{8}$. Some part of washed root samples after drying in oven, finely grinded for evaluation of changes in chemical constituents related to mycoflora. Quantitative estimation of chemical constituents was carried out from first day to $90^{\text {th }}$ day of incubation. Chemical analysis were estimated by the procedure described by Lowry et $a l^{6}$ for total protein, Singh et $a l^{12}$ for total phenols, Harborne ${ }^{3}$ for total alkaloids and Kokate et $a l^{5}$ for glycosides. Anthrone methods for Total sugars (TS) and Dinitrosalicilic acid (DNSA) method for Reducing sugar (RS) amount ${ }^{11}$ were also followed. Simple correlation were run between selected parameters using Statistical Package for Social Science (SPSS) software in which statistical significance was determined at $0.05 \%$ probability levels 
M Rashidi et al / IJRAP 3(6), Nov-Dec 2012

Table 1: Percentage incidence of fungal isolated from the market root of Gmelina arborea stored at various relative humidity

\begin{tabular}{|c|c|c|c|c|c|c|c|c|c|c|c|c|c|c|c|c|c|c|c|c|c|}
\hline \multirow[t]{2}{*}{ Mycoflora } & \multirow[t]{2}{*}{ con } & \multicolumn{4}{|c|}{$30 \%$} & \multicolumn{4}{|c|}{$50 \%$} & \multicolumn{4}{|c|}{$75 \%$} & \multicolumn{4}{|c|}{$96 \%$} & \multicolumn{4}{|c|}{$100 \%$} \\
\hline & & 15 & 30 & 45 & 60 & 15 & 30 & 45 & 60 & 15 & 30 & 45 & 60 & 15 & 30 & 45 & 60 & 15 & 30 & 45 & 60 \\
\hline Aspergillus niger & 0.57 & 0.57 & 0.57 & 0.76 & 0.95 & 0.38 & 0.57 & 0.95 & 1.71 & 0.95 & 1.33 & 1.71 & 2.09 & 0.38 & 0.57 & 0.95 & 1.52 & 0.95 & 1.71 & 2.85 & 5.71 \\
\hline A. flavus & 0.19 & 0.38 & 0.38 & 0.57 & 0.76 & 0.19 & 0.57 & 1.33 & 1.90 & 0.19 & 0.38 & 0.57 & 0.76 & 0.57 & 0.76 & 0.95 & 1.52 & 0.57 & 0.76 & 1.33 & 2.85 \\
\hline A. fumigatus & - & - & - & - & 0.19 & - & - & 0.19 & 0.19 & - & 0.19 & 0.38 & 0.57 & 0.19 & 0.57 & 0.76 & 1.14 & 0.38 & 0.57 & 1.33 & 1.71 \\
\hline A. parasiticus & - & - & - & - & 0.19 & - & - & 0.19 & 0.19 & - & - & 0.19 & 0.38 & - & 0.19 & 0.19 & 0.38 & - & 0.38 & 0.57 & 0.76 \\
\hline A. tamarii & - & - & - & - & 0.19 & - & - & - & 0.19 & - & - & - & 0.19 & - & - & 0.19 & 0.19 & - & 0.19 & 0.19 & 0.38 \\
\hline A. terreus & - & - & - & - & 0.19 & - & - & 0.19 & 0.19 & - & - & 0.19 & 0.19 & - & 0.19 & 0.19 & 0.38 & - & 0.38 & 0.57 & 0.76 \\
\hline Penicillium sp. & - & - & - & - & - & - & - & - & - & - & - & - & - & - & - & - & 0.19 & - & - & 0.19 & 0.38 \\
\hline Trichoderma sp. & 0.19 & 0.19 & 0.19 & 0.19 & 0.38 & 0.19 & 0.19 & 0.38 & 0.67 & 0.19 & 0.19 & 0.57 & 0.76 & 0.19 & 0.38 & 0.57 & 0.95 & 0.57 & 0.95 & 1.33 & 2.095 \\
\hline Fusarium solani & - & - & - & - & - & - & - & - & 0.19 & - & - & 0.19 & 0.19 & - & - & 0.19 & 0.38 & - & 0.57 & 0.76 & 1.33 \\
\hline F. oxysporum & - & - & - & - & 0.19 & - & - & - & 0.19 & - & - & - & 0.19 & - & - & - & 0.19 & - & 0.19 & 0.38 & 0.57 \\
\hline $\begin{array}{c}\text { Scytallidium } \\
\text { thermophilum }\end{array}$ & - & - & - & - & 0.19 & - & - & 0.19 & 0.19 & - & - & 0.19 & 0.38 & - & - & 0.19 & 0.38 & - & 0.38 & 0.57 & 1.33 \\
\hline Alternaria sp. & - & - & - & - & - & - & - & - & - & - & - & - & 0.19 & - & - & - & 0.19 & - & 0.19 & 0.38 & 0.57 \\
\hline Curvularia lunata & - & - & - & 0.19 & 0.38 & - & - & 0.19 & 0.38 & - & 0.19 & 0.38 & 0.57 & - & 0.19 & 0.38 & 0.57 & 0.38 & 0.57 & 0.95 & 1.33 \\
\hline Chaetomium sp. & - & - & - & - & - & - & - & - & - & - & - & - & 0.19 & - & - & 0.19 & 0.38 & - & 0.19 & 0.38 & 0.57 \\
\hline Monilia sitophila & - & - & - & - & - & - & - & - & - & - & - & - & - & - & - & - & - & - & - & 0.19 & 0.38 \\
\hline Total & 0.95 & 1.14 & 1.14 & 1.71 & 3.61 & 0.76 & 0.76 & 3.61 & 5.99 & 1.33 & 2.28 & 4.37 & 6.65 & 2.28 & 2.85 & 4.75 & 9.31 & 2.85 & 7.03 & 11.97 & 20.72 \\
\hline
\end{tabular}

Table 2: Deterioration of Total sugar (TS) and reducing sugar (RS) content (mg/100mg) in root Gmelina arborea at different relative humidities

\begin{tabular}{|c|c|c|c|c|c|c|c|c|c|c|c|c|}
\hline \multirow{2}{*}{$\begin{array}{c}\text { Incubation } \\
\text { days }\end{array}$} & \multicolumn{2}{|c|}{ control } & \multicolumn{2}{|c|}{$30 \%$} & \multicolumn{2}{|c|}{$50 \%$} & \multicolumn{2}{|c|}{$75 \%$} & \multicolumn{2}{|c|}{$96 \%$} & \multicolumn{2}{|c|}{$100 \%$} \\
\hline & TS & RS & TS & RS & TS & RS & TS & RS & TS & RS & TS & RS \\
\hline 1 day & $\begin{array}{c}49.53 \\
\pm 0.072\end{array}$ & $\begin{array}{c}22.62 \\
\pm 16.14\end{array}$ & $\begin{array}{c}49.53 \\
\pm 0.072\end{array}$ & $\begin{array}{c}22.62 \\
\pm 16.14\end{array}$ & $\begin{array}{c}49.53 \\
\pm 0.072\end{array}$ & $\begin{array}{c}22.62 \\
\pm 16.14\end{array}$ & $\begin{array}{c}49.53 \\
\pm 0.072\end{array}$ & $\begin{array}{c}22.62 \\
\pm 16.14\end{array}$ & $\begin{array}{c}49.53 \\
\pm 0.072\end{array}$ & $\begin{array}{c}22.62 \\
\pm 16.14\end{array}$ & $\begin{array}{c}49.53 \\
\pm 0.072\end{array}$ & $\begin{array}{c}22.62 \\
\pm 16.14\end{array}$ \\
\hline 30 days & $\begin{array}{c}49.53 \\
\pm 0.072 \mathrm{e}\end{array}$ & $\begin{array}{c}22.62 \\
\pm 16.14 \mathrm{e}\end{array}$ & $\begin{array}{c}48.65 \\
\pm 0.56 \mathrm{~d}\end{array}$ & $\begin{array}{c}21.71 \\
\pm 16.19 \mathrm{~d}\end{array}$ & $\begin{array}{c}48.10 \\
\pm 0.70 \mathrm{c}\end{array}$ & $\begin{array}{c}21.26 \\
\pm 14.95 \mathrm{~cd}\end{array}$ & $\begin{array}{l}77.011 \\
\pm 0.40 \mathrm{~b}\end{array}$ & $\begin{array}{c}20.81 \\
\pm 15.15 \mathrm{~b}\end{array}$ & $\begin{array}{c}46.63 \\
\pm 0.19 \mathrm{ab}\end{array}$ & $\begin{array}{c}18.09 \\
\pm 15.37\end{array}$ & $\begin{array}{l}45.41 \\
\pm 1.14\end{array}$ & $\begin{array}{c}15.74 \\
\pm 13.99 \mathrm{a}\end{array}$ \\
\hline 45 days & $\begin{array}{c}49.53 \\
\pm 0.072 \mathrm{e}\end{array}$ & $\begin{array}{c}22.62 \\
\pm 16.14 \mathrm{e}\end{array}$ & $\begin{array}{c}47.72 \\
\pm 0.56 \mathrm{~d}\end{array}$ & $\begin{array}{c}19.90 \\
\pm 17.08 \mathrm{~d}\end{array}$ & $\begin{array}{c}47.13 \\
\pm 0.26 \mathrm{c}\end{array}$ & $\begin{array}{c}19.45 \\
\pm 15.15 \mathrm{c}\end{array}$ & $\begin{array}{c}46.17 \\
\pm 0.26 \mathrm{~b}\end{array}$ & $\begin{array}{c}19.004 \\
\pm 14.71 \mathrm{~b}\end{array}$ & $\begin{array}{c}45.48 \\
\pm 0.98 \mathrm{ab}\end{array}$ & $\begin{array}{c}16.74 \\
\pm 12.88 \mathrm{ab}\end{array}$ & $\begin{array}{c}44.61 \\
\pm 0.72 \mathrm{ab}\end{array}$ & $\begin{array}{c}13.57 \\
\pm 13.05 \mathrm{a}\end{array}$ \\
\hline 75 days & $\begin{array}{c}49.53 \\
\pm 0.072 \mathrm{e}\end{array}$ & $\begin{array}{c}22.62 \\
\pm 16.14 \mathrm{e}\end{array}$ & $\begin{array}{c}45.74 \\
\pm 0.89 \mathrm{~d}\end{array}$ & $\begin{array}{c}14.47 \\
\pm 15.43 \mathrm{~d}\end{array}$ & $\begin{array}{c}45.91 \\
\pm 0.69 \mathrm{c}\end{array}$ & $\begin{array}{c}14.47 \\
\pm 13.73 \mathrm{c}\end{array}$ & $\begin{array}{c}43.39 \\
\pm 0.69 \mathrm{~b}\end{array}$ & $\begin{array}{c}14.02 \\
\pm 10.65 \mathrm{~b}\end{array}$ & $\begin{array}{c}41.32 \\
\pm 0.69 \mathrm{a}\end{array}$ & $\begin{array}{c}13.12 \\
\pm 10.48 \mathrm{a}\end{array}$ & $\begin{array}{l}39.89 \\
\pm 0.57\end{array}$ & $\begin{array}{c}11.76 \\
\pm 8.84 \mathrm{a}\end{array}$ \\
\hline 90 days & $\begin{array}{c}49.53 \\
\pm 0.072 \mathrm{e}\end{array}$ & $\begin{array}{c}22.62 \\
\pm 16.14 \mathrm{e}\end{array}$ & $\begin{array}{c}45.07 \\
\pm 0.76 \mathrm{~cd}\end{array}$ & $\begin{array}{c}13.57 \\
\pm 14.31 \mathrm{~d}\end{array}$ & $\begin{array}{c}43.81 \\
\pm 0.57 \mathrm{c}\end{array}$ & $\begin{array}{c}13.57 \\
\pm 13.53 \mathrm{c}\end{array}$ & $\begin{array}{c}42.63 \\
\pm 0.83 \mathrm{~b}\end{array}$ & $\begin{array}{c}12.66 \\
\pm 9.96 \mathrm{~b}\end{array}$ & $\begin{array}{c}40.10 \\
\pm 0.38 \mathrm{a}\end{array}$ & $\begin{array}{c}11.31 \\
\pm 9.55 \mathrm{a}\end{array}$ & $\begin{array}{c}38.69 \\
\pm 0.69 \mathrm{a}\end{array}$ & $\begin{array}{c}11.28 \\
\pm 0.62 \mathrm{a}\end{array}$ \\
\hline
\end{tabular}

Data in tables and are the mean of three replicates \pm standard deviation. P- Value denoted the significance of differences between the mean by univariate comparison statistics. The value followed by different letters differ significantly by Duncan's multiple rang test at $\mathrm{P}=\mathrm{Sig}=0.05$ 
Table 3: Deterioration of protein content $(\mathrm{mg} / \mathbf{1 0 0} \mathrm{mg})$ in root of Gmelina arborea at different relative humidities

\begin{tabular}{|c|c|c|c|c|c|c|}
\hline Incubation days & control & $30 \%$ & $50 \%$ & $75 \%$ & $96 \%$ & $100 \%$ \\
\hline 1 day & $29.02 \pm 0.86$ & $29.02 \pm 0.86$ & $29.02 \pm 0.86$ & $29.02 \pm 0.86$ & $29.02 \pm 0.86$ & $29.02 \pm 0.86$ \\
\hline 15 days & $29.02 \pm 0.86 \mathrm{~d}$ & $28.75 \pm 0.78 \mathrm{~cd}$ & $28.05 \pm 0.60 \mathrm{~d}$ & $27.77 \pm 0.040 \mathrm{bc}$ & $26.66 \pm 0.65 \mathrm{a}$ & $26.25 \pm 0.21 \mathrm{a}$ \\
\hline 30days & $29.02 \pm 0.86 \mathrm{~d}$ & $27.91 \pm 0.36 \mathrm{~cd}$ & $27.50 \pm 0.21 \mathrm{~cd}$ & $26.38 \pm 0.71 \mathrm{bc}$ & $25.13 \pm 0.13 \mathrm{a}$ & $24.72 \pm 0.27 \mathrm{a}$ \\
\hline 45 days & $29.02 \pm 0.86 \mathrm{~d}$ & $27.36 \pm 0.13 \mathrm{~cd}$ & $25.97 \pm 0.28 \mathrm{~cd}$ & $25 \pm 0.036 \mathrm{bc}$ & $23.61 \pm 0.21 \mathrm{a}$ & $23.05 \pm 0.13 \mathrm{a}$ \\
\hline 60 days & $29.02 \pm 0.86 \mathrm{~d}$ & $26.38 \pm 0.24 \mathrm{~cd}$ & $25.13 \pm 0.44 \mathrm{~cd}$ & $24.30 \pm 0.76 \mathrm{~b}$ & $23.33 \pm 0.28 \mathrm{a}$ & $22.22 \pm 1.04 \mathrm{a}$ \\
\hline 75 days & $29.02 \pm 0.86 \mathrm{~d}$ & $25 \pm 0.036 \mathrm{~cd}$ & $23.61 \pm 0.21 \mathrm{c}$ & $22.22 \pm 0.34 \mathrm{~b}$ & $20.97 \pm 0.52 \mathrm{a}$ & $20.41 \pm 0.90 \mathrm{a}$ \\
\hline 90 days & $29.02 \pm 0.86 \mathrm{~d}$ & $23.75 \pm 0.86 \mathrm{c}$ & $22.22 \pm 0.34 \mathrm{c}$ & $20.83 \pm 0.65 \mathrm{~b}$ & $19.86 \pm 0.57 \mathrm{a}$ & $19.02 \pm 0.71 \mathrm{a}$ \\
\hline
\end{tabular}

Data in tables and are the mean of three replicates \pm standard deviation. P- Value denoted the significance of differences between the mean by univariate comparison statistics. The value followed by different letters differ significantly by Duncan's multiple rang test at $\mathrm{P}=\mathrm{Sig}=0.05$

Table 4: Deterioration of total phenols content $(\mathrm{mg} / \mathbf{1 0 0} \mathrm{mg})$ in root of Gmelina arborea at different relative humidities

\begin{tabular}{|c|c|c|c|c|c|c|}
\hline Incubation days & control & $30 \%$ & $50 \%$ & $75 \%$ & $96 \%$ & $100 \%$ \\
\hline 1 day & $4.05 \pm 0.070$ & $4.05 \pm 0.070$ & $4.05 \pm 0.070$ & $4.05 \pm 0.070$ & $4.05 \pm 0.070$ & $4.05 \pm 0.070$ \\
\hline 15days & $4.05 \pm 0.070 \mathrm{~d}$ & $3.97 \pm 0.022 \mathrm{~cd}$ & $3.93 \pm 0.011 \mathrm{~cd}$ & $3.79 \pm 0.052 \mathrm{bc}$ & $3.74 \pm 0.011 \mathrm{ab}$ & $3.62 \pm 0.030 \mathrm{a}$ \\
\hline 30days & $4.05 \pm 0.070 \mathrm{~d}$ & $3.91 \pm 0030 \mathrm{c}$ & $3.81 \pm 0.30 \mathrm{c}$ & $3.68 \pm 0.34 \mathrm{~b}$ & $3.54 \pm 0.090 \mathrm{ab}$ & $3.40 \pm 0.049 \mathrm{a}$ \\
\hline 45 days & $4.05 \pm 0.070 \mathrm{~d}$ & $3.74 \pm 0.079 \mathrm{c}$ & $3.58 \pm 0.070 \mathrm{c}$ & $3.40 \pm 0.090 \mathrm{~b}$ & $3.28 \pm 0.010 \mathrm{a}$ & $3.07 \pm 0.074 \mathrm{a}$ \\
\hline 60 days & $4.05 \pm 0.070 \mathrm{~d}$ & $3.68 \pm 0.099 \mathrm{c}$ & $3.46 \pm 0.090 \mathrm{c}$ & $3.30 \pm 0.11 \mathrm{~b}$ & $3.01 \pm 0.15 \mathrm{a}$ & $2.81 \pm 0.079 \mathrm{a}$ \\
\hline 75 days & $4.05 \pm 0.070 \mathrm{~d}$ & $3.54 \pm 0.15 \mathrm{c}$ & $3.36 \pm 0.069 \mathrm{c}$ & $3.14 \pm 0.13 \mathrm{~b}$ & $2.95 \pm 0.17 \mathrm{a}$ & $2.71 \pm 0.30 \mathrm{a}$ \\
\hline 90 days & $4.05 \pm 0.070 \mathrm{~d}$ & $3.34 \pm 0.12 \mathrm{c}$ & $3.16 \pm 0.030 \mathrm{c}$ & $3.01 \pm 0.13 \mathrm{~b}$ & $2.86 \pm 0.19 \mathrm{a}$ & $2.55 \pm 0.16 \mathrm{a}$ \\
\hline
\end{tabular}

Data in tables and are the mean of three replicates \pm standard deviation. P- Value denoted the significance of differences between the mean by univariate comparison statistics. The value followed by different letters differ significantly by Duncan's multiple rang test at $\mathrm{P}=\mathrm{Sig}=0.05$

\begin{tabular}{|c|c|c|c|c|c|c|}
\hline $\begin{array}{c}\text { Incubation } \\
\text { days }\end{array}$ & control & $30 \%$ & $50 \%$ & $75 \%$ & $96 \%$ & $100 \%$ \\
\hline 1 day & $25.53 \pm 1.14$ & $25.53 \pm 1.14$ & $25.53 \pm 1.14$ & $25.53 \pm 1.14$ & $25.53 \pm 1.14$ & $25.53 \pm 1.14$ \\
\hline 15 days & $25.52 \pm 1.024 \mathrm{e}$ & $25.52 \pm 1.14 \mathrm{~d}$ & $25.51 \pm 0.094 c$ & $25.50 \pm 1.011 \mathrm{c}$ & $25.49 \pm 1.25 \mathrm{ab}$ & $25.47 \pm 1.04 \mathrm{a}$ \\
\hline 30days & $25.50 \pm 1.04 \mathrm{e}$ & $25.49 \pm 1.14 \mathrm{~d}$ & $25.44 \pm 1.17 \mathrm{c}$ & $25.39 \pm 0.13 c$ & $25.32 \pm 1.11 \mathrm{ab}$ & $25.28 \pm 1.12 \mathrm{a}$ \\
\hline 45 days & $25.48 \pm 1.038 \mathrm{e}$ & $25.44 \pm 1.070 \mathrm{~d}$ & $25.39 \pm 0.19 \mathrm{c}$ & $25.28 \pm 1.15 b c$ & $25.20 \pm 1.068 \mathrm{a}$ & $25.15 \pm 1.26 \mathrm{a}$ \\
\hline 60 days & $25.46 \pm 1.047 \mathrm{e}$ & $25.39 \pm 1.072 \mathrm{~cd}$ & $25.32 \pm 1.10 \mathrm{c}$ & $25.17 \pm 1.17 b c$ & $25.086 \pm 1.01 \mathrm{a}$ & $24.96 \pm 1.07 \mathrm{a}$ \\
\hline 75 days & $25.46 \pm 1.035 \mathrm{e}$ & $25.29 \pm 1.064 \mathrm{~cd}$ & $25.21 \pm 1.10 \mathrm{c}$ & $25.04 \pm 1.17 \mathrm{bc}$ & $24.91 \pm 1.11 \mathrm{a}$ & $24.79 \pm 1.12 \mathrm{a}$ \\
\hline 90 days & $25.45 \pm 1.034 \mathrm{e}$ & $25.16 \pm 1.092 \mathrm{~cd}$ & $25.08 \pm 1.048 b c$ & $24.96 \pm 1.076 b$ & $24.82 \pm 1.094 a$ & $24.68 \pm 1.14 a$ \\
\hline
\end{tabular}

Data in tables and are the mean of three replicates \pm standard deviation. P- Value denoted the significance of differences between the mean by univariate comparison statistics. The value followed by different letters differ significantly by Duncan's multiple rang test at $\mathrm{P}=\mathrm{Sig}=0.05$

\begin{tabular}{|c|c|c|c|c|c|c|}
\hline $\begin{array}{c}\text { Incubation } \\
\text { days }\end{array}$ & control & $30 \%$ & $50 \%$ & $75 \%$ & $96 \%$ & $100 \%$ \\
\hline 1 day & $16.71 \pm 0.090$ & $16.71 \pm 0.090$ & $16.71 \pm 0.090$ & $16.71 \pm 0.090$ & $16.71 \pm 0.090$ & $16.71 \pm 0.090$ \\
\hline 15days & $16.71 \pm 0.090 \mathrm{~d}$ & $16.71 \pm 0.097 \mathrm{~cd}$ & $16.71 \pm 0.10 \mathrm{c}$ & $16.70 \pm 0.095 \mathrm{bc}$ & $16.69 \pm 0.010 \mathrm{ab}$ & $16.68 \pm 0.10 \mathrm{a}$ \\
\hline 30days & $16.71 \pm 0.090 \mathrm{~d}$ & $16.71 \pm 0.087 \mathrm{~cd}$ & $16.69 \pm 0.10 \mathrm{c}$ & $16.68 \pm 0.10 \mathrm{c}$ & $16.62 \pm 0.10 \mathrm{ab}$ & $16.62 \pm 0.015 \mathrm{a}$ \\
\hline 45 days & $16.69 \pm 0.010 \mathrm{~d}$ & $16.68 \pm 0.010 \mathrm{~cd}$ & $16.67 \pm 0.13 c$ & $16.61 \pm 0.090 \mathrm{bc}$ & $16.56 \pm 0.12 \mathrm{a}$ & $16.55 \pm 0.12 \mathrm{a}$ \\
\hline 60 days & $16.80 \pm 0.023 \mathrm{~d}$ & $16.64 \pm 0.10 \mathrm{~cd}$ & $16.61 \pm 0.11 \mathrm{c}$ & $16.57 \pm 0.060 \mathrm{bc}$ & $16.50 \pm 0.10 \mathrm{a}$ & $16.42 \pm 0.05 \mathrm{a}$ \\
\hline 75 days & $16.69 \pm 0.10 \mathrm{~d}$ & $16.58 \pm 0.10 \mathrm{c}$ & $16.53 \pm 0.070 \mathrm{c}$ & $16.50 \pm 0.076 \mathrm{bc}$ & $16.42 \pm 0.066 \mathrm{a}$ & $16.36 \pm 0.041 \mathrm{a}$ \\
\hline 90 days & $16.68 \pm 0.017 \mathrm{~d}$ & $16.55 \pm 0.10 \mathrm{c}$ & $16.50 \pm 0.11 \mathrm{c}$ & $16.38 \pm 0.03 \mathrm{~b}$ & $16.31 \pm 0.01 \mathrm{a}$ & $16.25 \pm 0.026 \mathrm{a}$ \\
\hline
\end{tabular}

Data in tables and are the mean of three replicates \pm standard deviation. P- Value denoted the significance of differences between the mean by univariate comparison statistics. The value followed by different letters differ significantly by Duncan's multiple rang test at $\mathrm{P}=\mathrm{Sig}=0.05$

\section{RESULTS}

Total 15 fungi from market roots of $G$. arborea were isolated at different relative humidities which include: Aspergillus niger, A. flavus, A. fumigatus, A. parasiticus, A. tamarii, A. terreus, Penicillium sp., Trichoderma sp., Fusarium solani, F. oxysporum, Scytallidium thermophilum, Alternaria sp., Curvularia lunata, Chaetomium sp. and Monilia sitophila. Total percentage incidence of fungi under 30, 50, 75, 96 and 100\% RH after 15 days of incubation observed were $1.14,0.76$, $1.33,2.85$ and $2.85 \%$. These percentages increased to $3.61,5.99,6.65,9.31$ and $20.72 \%$ after 60 days of incubation (Table 1).

The drug stored under higher relative humidities $(75,96$ and $100 \%$ ) observed maximum reduction in chemical constituents while percentage incidence of the fungi were also maximum.

After 15 days of incubation period, under 30, 50, 75, 96 and $100 \% \mathrm{RH}$, amounts of Total sugar (TS) and Reducing sugar (RS) recorded 49.49, 22.62; 49.24, 22.62\%; 49.07,
$22.17 \% ; 48.48,21.26 \%$ and $48.27,20.81 \%$ respectively, these amounts deteriorated to $45.07,13.57 \%(30 \% \mathrm{RH})$; 43.81, 13.57\% (50\% RH); 42.63, 12.66\% (75\% RH); $40.1011 .31 \%(96 \% \mathrm{RH})$ and $38.69,11.29 \%(100 \% \mathrm{RH})$ after 90 days of incubation (Table 2).

In case of total protein amount, under 30, 50, 75, 96 and $100 \% \mathrm{RH}$ after 15 days of storage, total value of protein recorded 28.75, 28.05, 27.77, 26.66 and $26.25 \%$ respectively, while theses values decreased to 23.75 , $22.22,20.83,19.86$ and $19.02 \%$ after 90 days of incubation (Table 3 ).

The control samples contained $4.72 \%$ of total phenol amount. After 15 days of incubation period in all tested relative humidity $30,50,75,96$ and $100 \%$ RH. Total phenol was recorded $3.97,3.93,3.79,3.74$ and $3.62 \%$. These values of total phenols reduced to $3.34,3.16,3.01$, 2.86 and $2.55 \%$ after 90 days of incubation period (Table 4).

At the first day of incubation control of market, sample showed $25.53 \%$ total alkaloids (Table 5). After 15 days of 
incubation and under 30, 50, 75, 96 and $100 \% \mathrm{RH}$, total amount of alkaloid; recorded 25.52, 25.51, 25.50, 25.49, $25.47 \%$; respectively. These values of total alkaloids deteriorated to $25.16,25.08,24.96,24.82$, and $24.68 \%$ after 90 days of incubation period. Deterioration of glycosides amounts also recorded, while after 15 days of storage the values of glycosides showed 16.71, 16.71, $16.70,16.69$ and $16.68 \%$ under 30, 50, 75, 96 and 100\% $\mathrm{RH}$, after 90 days of incubation period, these amounts of glycosides decreased to $16.55,16.50,16.38,16.31$ and $16.25 \%$ under different relative humidity, respectively (Table 6).

The result were tested statistically which showed the effect of relative humidity and incubation days in reduction of sugar, proteins, phenols, alkaloids and glycosides contents were significant at $5 \%$ level of significance $(\mathrm{P}$ value $<0.05)$.

\section{DISCUSSION}

Fungal activity can cause changes during storage of herbal plants and their products that are detrimental to nutritive value. Specifically, nutrients are lost because of changes in carbohydrates, protein, phenols and other constituents. Conditions that favour for fungal activity lead to carbohydrate decomposition. Sugars are consumed and converted into $\mathrm{CO}_{2}$ and $\mathrm{H}_{2} \mathrm{O}$. Reduction of protein contents maybe is due to fungi and their utilization, photolytic enzymes produced by fungi can modify the proteins in tissues by hydrolyzing them into polypeptides and amino acids. Function of phenols and some classes of phenolic compounds are well established. For examples lignins which are as structural material of the cell walls, but generally they may be natural resistance factors for reduction of phenol amount during pathogenesis and it's subsequently utilization as carbon source by fungi for their growth. Experimental result revealed that there was gradual depletion in alkaloids and glycosides concentration under different relative humidity which might be due to its degradation into simple form by microbial enzymes and utilization of them by fungi.

\section{ACKNOWLEDGMENT}

Authors would like to express a sincere thank to Head, Department of Botany, University of Pune, India for encouragement and necessary laboratory facilities.

\section{REFERENCES}

1. Asokar LV, Kakkar KK and Chankre OJ. Second Supplement to Glossary of Indian Medicinal Plants With Active Principles. Part I. CSIR, 1992.

2. Barnet HL, Hunter BB. Illustrated Genera of Imperfect Fungi. Minneapolis Burgress Publishing Company. Minneapolis. 1972. PMCid:1034928

3. Harborne JB. Phytochemical methods -London. New York, Chapman \& Hall, 1973; 39-42.

4. International seed testing association, International rules for seed health testing, Proc. International seed test assoc. 1966; 31:1.

5. Kokate CK, Gokhale SB, Purohit AP. Pharmacognosy. Nirali Prakashan Pune, 2002.

6. Lowry $\mathrm{OH}$, Rosebrough NJ, Farr AL, Randall K, Protein measurement with folin phenol reagent. J. Biol. Chemistry. 1951; $193: 256-275$.

7. Nadkarni AK. Indian Materia Medica. 3th edition Dhootapapeshwar, Prakashan. 1951; 1319.

8. Nelson PE, Tossoum TA, Marasas WFO. Fusarium Species, An Illustrated Mannual for Identification. The Pennsylvania State University Press. U. S. A. 1983; 193. PMid:6294277

9. Raper KB, Thomas C. A manual of Penicillia Williams and Wilkins. Battimore 1949; 850 .

10. Roy AK. Mycological problems of crude drugs - overview and challenges. Indian Phytopathology, 2003; 56,1-13.

11. Sadasivam S, Manickam A. Biochemical Methods of Agricultural Sciences. Wiley Estern Ltd, New Delhi, India, 1992.

12. Singh M, Singh SS, Sanwal GG. A new calorimetric method for the determination of phenolics. Ind. Phytopath, 1978; 16: $712-714$.

13. Wallace HAH, Sinha RN, Mills JJ. Fungi associated with small wheat bulks during prolonged storage in Manitoba. Can. J. Bot. 1976; 54, 1332-1343. http://dx.doi.org/10.1139/b76-146

14. Warrier PK, Nambiar VPK, Ramankutty C. Indian Medicinal Plants. Vol 1-5 Orient longman Ltd., Madras, 1995.

15. Wexler A, Hasegawa S. Relative humidity and temperature relationship of some saturated salt solutions in the temperature range 0 to $50^{0 \mathrm{c}}$ J. Res. Nat. Bur. Satnd. 1954; 53: 19-26. http://dx.doi.org/10.6028/jres.053.003

Cite this article as:

M Rashidi and SS Deokule. Biodeterioration of chemical constituents in drug Gmelina arborea Linn. under storage of different relative humidities due to spoilage of fungi. Int. J. Res. Ayur. Pharm. 2012; 3(6):818-821 